\title{
La identidad de la empresa social en España: análisis desde cuatro realidades socioeconómicas
}

\author{
Marta Solórzano García \\ Carmen Guzmán Alfonso \\ Teresa Savall Morera \\ Esther Villajos Girona
}

RESUMEN: La realidad y la literatura científica de la empresa social han ido adquiriendo mayor relevancia durante los últimos años en España, aunque no ha llegado a equipararse a los niveles de otros países europeos, en los cuales el concepto goza de un mayor reconocimiento y desarrollo. Si bien se pueden identificar ejemplos de empresas sociales en nuestro país desde el siglo XV, no es hasta hace una década cuando se ha acuñado el concepto de manera más generalizada.

Nuestro objetivo es identificar los modelos de empresa social existentes en España sobre cada uno de los enfoques considerados en la literatura específica de empresa social.

Partiendo de la metodología y el trabajo desarrollado dentro del proyecto ICSEM (equipo español), estableceremos una clasificación de los distintos modelos de empresas sociales, utilizando como referencia las fronteras conceptuales de cuatro realidades socioeconómicas presentes en España: la economía social, el Tercer Sector de Acción Social, la economía solidaria y el Emprendimiento Social.

Como resultado más relevante obtenemos una nueva perspectiva de análisis de las empresas sociales, contribuyendo así al debate actual de delimitación del concepto y realidad de la empresa social, en España en particular, y en la esfera académica en general.

PALABRAS CLAVE: Empresa social, economía social, Tercer Sector de Acción Social, economía solidaria, emprendimiento social.

CLAVES ECONLIT: L31, A13, J54, P51.

Cómo citar este artículo / How to cite this article: SOLÓRZANO, M., GUZMÁN, C., SAVALL, T. \& VILLAJOS,

E. (2018): "Identidad de la empresa social en España: análisis desde cuatro realidades socioeconómicas", CIRIEC-España, Revista de Economía Pública, Social y Cooperativa, 92, 155-182.

Correspondencia: Marta Solórzano García (correspondencia), Profesora Titular de Universidad, Departamento de Organización de Empresas, Universidad Nacional de Educación a Distancia, UNED; msolorzano@ cee.uned.es; Carmen Guzmán Alfonso, Profesora Ayudante Doctor, Departamento Economía Aplicada I, Universidad de Sevilla, cguzman2@us.es; Teresa Savall Morera, Profesora Ayudante Doctor, Departamento Economía Aplicada, Universidad de Valencia, teresa.savall@uv.es; Esther Villajos Girona, Investigadora, IDOCALDepartamento de Psicología Social, Universidad de Valencia, esther.villajos@uv.es. 


\section{EXPANDED ABSTRACT}

\section{The identity of the social entreprise in Spain: analysis from four different socioeconomic realities}

Scientific literature about Social Enterprises has become more relevant during the last years in Spain; even if it is not the same level of discussion as in other European countries in which the concept enjoys greater recognition and development. Although some examples of social enterprises can be identified in Spain since the fifteenth century, it is not until the last decade when the concept gained more popularity.

The objective of this paper is to analyze the reality of social enterprises in Spain and propose a typology of the Spanish social enterprise. To do so, EMES ideal definition of Social Enterprise has been taken as a reference to look at Spanish Social Enterprise in four socioeconomic realities: Social Economy, Third Sector of Social Action, Solidarity Economy and Social Entrepreneurship.

In order to achieve this objective, the work and methodology previously developed by the ICSEM Project is used as a starting point. The project aims to compare Social Enterprise models and their respective institutionalisation' processes across the world. In our case, although we will also include the dimensions of the EMES' ideal type of Social Enterprise, we will focus in Spain and use a different theoretical approach, more related to the socioeconomic framework in the target territory of study.

A literature review has been developed, focused on analyzing and describing the concept of social enterprise internationally and nationally. As a result, two main ideas have been identified: 1) There is no agreement on the definition of social enterprises at both levels. This is a consequence of the number and diversity of entities and business' organizations that co-exist under this concept, although different motivations and characteristics can be distinguished among them. 2) Taking into account the different conceptualizations of social enterprises, some authors (e.g. Dees \& Anderson, 2006; Defourny \& Nyssens, 2012) distinguish among two major schools of thought: the European and the Anglo-Saxon. These two schools use different approaches to study the social enterprise reality. The European school distinguish between social economy and solidarity economy approaches, whereas the Anglo-Saxon school uses the income generation approach (or Third Sector of Social Action) and the social entrepreneurship one.

Second, as result of our analysis, a matrix is proposed. The columns of the matrix are the indicators of the three dimensions (social, governmental and economic) of EMES' ideal type of 
social entreprise definition. The files in the matrix correspond to the different Spanish socioeconomic realities and the social enterprises that exist in them.

According to the obtained results, the following conclusions can be extracted. Firstly, social enterprises that are embedded in the market social economy, clearly fulfill the criteria from the economic and social dimensions. The governance criteria will depend of the ownership type of these organizations. Cooperatives, by its nature, adopt a participative and democratic decision making process, and in the case of WISE (Work Integration Social Entreprise) and CEE (in Spanish Centros Especiales deEmpleo), governance will depend on their managers' intentions, which is not strange, taking into consideration their particular characteristics and their workers situation.

The Spanish Law of Social Economy (2011) refers in its preamble to the Charter of Principles of the social economy from CEP-CMAF ${ }^{i}$, and in this same sense, Monzón (2006) considers intern democracy to be a conclusive variable in order to include a non-for-profit organization into the Social Economy. Therefore, when considering social enterprises from the Social Economy' perspective, the governance criteria is the decisive one, besides the limitation of profits.

However, and secondly, from the solidarity economy, debate on social change, the construction of alternative economic models, alternatives to capitalism or ways of combating poverty and social exclusion are the central focus of thought and reflection. Social enterprise is conceived as part of a social movement including organizations and activities that generate a solidarity and alternative thought and practice. Thus, besides the importance of the social and governance dimensions, when analyzed within the context of solidarity economy, is its social transformation mission, understood as a political implication, or the search for an economic and social paradigm change, what makes the difference in social enterprises.

Thirdly, organizations that emerge from the Third Sector of Social Action are characterized by their high compliance with the criteria of social dimension and governance. The economic indicators are met with lower intensity, depending on their market orientation extent. The fulfillment of the social dimension is justified by two characteristics: their clear orientation towards the general interest (as they are organizations of general interest by definition), and by being under the principle of non-distribution of benefits. The governance criteria and its democratic character is met by associations (because of their associative nature). In the case of foundations, it will depend on the will of its managers.

And fourthly, social enterprises from the social entrepreneurship sector are conceived as a business to resolve a social problem as a purpose. The main difference from the rest of social enterprises 
is that their market orientation (their identity as a business) is really strong and this make the difference when comparing with the rest. The fulfillment of the social dimension will depend on the extent to which the social objective is accomplished, as the ownership type does not guarantee it, and there is no limitation for profit sharing. The fulfillment of the governance dimension, as well as the social dimension, will depend on the will of the entrepreneurs, since nothing prevents the decision from being based on the capital ownership.

Our analysis led to the recognition of different models of social enterprise in Spain depending on the socio-economic reality it is studied from, with continuities and ruptures. Each model emphasizes a particular aspect of the social enterprise and represents the way it looks at it and the priorities it implicitly proposes for its development.

The concept, in that sense, from a methodological point of view, should go hand in hand with the reality that wants to represent. It is essential to introduce a dynamic character to the concept, if the latter is to define the former.

KEYWORDS: Social Enterprise, Social Economy, Third Sector of Social Action, Solidarity Economy, Social Entrepreneurship. 


\section{Introducción}

El interés por las empresas sociales por parte de los académicos, políticos, organizaciones profesionales y la sociedad civil ha crecido exponencialmente en los últimos años. Este incremento se debe principalmente a que este tipo de entidades se han convertido en una realidad contrastada para solucionar los problemas sociales, económicos y medioambientales que los Estados de bienestar no han sido capaces de solucionar dentro del contexto de crisis económica actual (Gonçalves, Carrara y Moro, 2016). A partir del año 2004 ha habido un incremento sin precedentes del número de citas de los términos "empresa social", "emprendimiento social" y "emprendedor social" en la literatura académica (Granados, et al., 2011).

Sin embargo, a pesar de la gran acogida que han tenido las empresas sociales tanto en el ámbito práctico como académico, cabe destacar que aún no existe una definición universalmente aceptada de empresa social (Bacq y Janssen, 2011; Deffourny y Nyssens, 2016; Villajos y Sanchis, 2013). No obstante, sí que existe el consenso de que dicho término designa a cualquier iniciativa empresarial cuyo objetivo es dar respuesta a una problemática social (Battilana, y Lee, 2014; Miller et al, 2012; Wry y York, 2017).

Por otro lado, existen en la literatura varios estudios que han intentado establecer unos modelos o tipologías de empresas sociales en función de diversos criterios. Alter (2007) contempla las empresas sociales bajo diferentes perspectivas y, siendo consciente de los límites borrosos que existen entre dichas perspectivas, distingue entre modelos fundamentales (modelo de apoyo al emprendedor, modelo de empleo, modelo dedicado al cliente de bajo ingreso, modelo cooperativo, entre otros), modelos combinados (complejos y mixtos) y modelos de reforzamiento (franquicia y socio privado-sin ánimo de lucro).

Defourny y Nyssens (2016) desarrollan un marco teórico en el que combinando la magnitud del colectivo beneficiado (público, mutuo o de capital) con el origen de los recursos identifican las trayectorias institucionales que generan cuatro principales modelos de empresas sociales: el modelo empresariales sin ánimo de lucro, el modelo de las cooperativas sociales, el modelo de los negocios sociales y el modelo de la empresa social del sector público.

Otros trabajos a destacar que han generado diversas clasificaciones de la empresa social sobre la base de su origen, propósito, trayectorias y discursos son los de Dees (2001), Spear et al. (2009), Teasdale (2012), Kerlin (2013) y Gordon (2015). 
A nivel nacional, establecer una clasificación de empresas sociales es aún una tarea pendiente, sobre todo teniendo en cuenta cómo pueden variar las realidades objeto de estudio de un territorio a otro y los distintos enfoques de los que se puede partir. No obstante se puede encontrar en la literatura algunos intentos de clasificación (Villajos y Sanchis, 2013). En este sentido destaca la realización de una primera aproximación dentro del marco del proyecto internacional ICSEM. En dicho trabajo, el equipo español llevó a cabo una modelización de la empresa social en el país en base a los sectores de actividad, llegando a unos resultados que no eran del todo concluyentes y que invitaban a nuevas investigaciones al respecto utilizando una metodología diferente (Millán-Foncea et al., 2017).

En este contexto, el objeto de la presente investigación es identificar los modelos de empresa social existentes en España bajo la concepción particular de cada uno de los enfoques considerados en la literatura específica de empresa social. De este modo, tomando como referencia de nuevo la definición de tipo ideal de empresa social definida por EMES, se parte de un marco teórico diferente, analizando la concepción y la evolución de la empresa social en España bajo cada una de estas cuatro perspectivas teóricas y definiendo los modelos en base a ellas. De esta manera, se pretende arrojar nuevas luces sobre la realidad de la empresa social en España.

\section{Marco teórico}

El hecho de que aún no exista una definición generalmente aceptada de empresa social radica en que existe una gran variedad de entidades y organizaciones empresariales que se engloban dentro de dicho concepto, pudiéndose distinguir diferentes motivaciones y especificidades entre dichas empresas. De esta forma, a la hora de afrontar el estudio de las mismas, resulta bastante difícil delimitar este campo de investigación.

No obstante, todas las definiciones proporcionadas en la literatura sobre empresa social tienen en común que en este término se engloban las iniciativas cuyo fin es el logro de una misión social mediante la utilización de mecanismos de mercado (Doherty, Haugh, y Lyon, 2014; Kerlin, 2009; Santos, Pache, y Birkholz, 2015). Su objetivo fundamental es la generación de valor social para los beneficiarios de su misión social y su fuente de ingresos principal es la vía comercial, dependiendo del mercado para su sostenibilidad financiera, de forma que las actividades comerciales se entienden como medios para obtener sus fines. Como tales, se conciben como organizaciones híbridas que combinan aspectos de las organizaciones benéficas y de las empresas comerciales (Battilana \& Lee, 2014; Besharov \& Smith, 2014; Mair, Mayer \& Lutz, 2015; Galaskiewicz \& Barringer, 2012)

A este respecto, y teniendo en cuenta las diferencias aportadas en las distintas contribuciones sobre empresa social, son varios los autores (Dees \& Anderson, 2006; Defourny \& Nyssens, 2012) 
que distinguen en la literatura dos grandes escuelas de pensamiento en el estudio de la misma: la europea y la anglosajona, en las cuales, a su vez, encontramos las diferentes realidades 0 enfoques en los que se centra la presente investigación para estudiar el fenómeno de la empresas sociales en España.

\subsection{Escuela Europea}

La escuela europea tiene sus raíces en la crisis de los ochenta y engloba a iniciativas que aparecen para proveer los servicios que el estado dejó de suministrar (Hoogendoorn et al., 2010). En esta escuela la unidad de observación es la empresa, con la peculiaridad de que su actividad está iniciada por un grupo de ciudadanos con un objetivo explícito de beneficio a la comunidad (Defourny \& Nyssens, 2012). Es decir, en Europa se parte de un esfuerzo colectivo para las iniciativas de emprendimiento social (Bacq \& Janssen, 2011). Dentro de esta escuela, podemos distinguir entre los enfoques o subescuelas de economía social y de economía solidaria.

\section{Enfoque de la economía social}

El enfoque de la economía social se refiere al grupo de entidades privadas que han sido creadas por sus miembros para la satisfacción de sus necesidades a través del mercado; cuyo funcionamiento está basado en principios distintos al de maximización de beneficios, tales como la priorización de las personas sobre el capital o la toma de decisiones de forma democrática; y que se rigen por valores como la solidaridad y el compromiso con el entorno (Glemain et al., 2013; Arrieta, 2014). De acuerdo con la literatura específica, se pueden distinguir dos subsectores diferentes dentro del sector de economía social: el subsector de mercado y el subsector de no mercado (Monzón \& Chaves, 2012; Bretos y Morandeira; 2016).

El primer grupo comprende aquellas entidades que obtienen sus recursos de ventas en el mercado y que pueden distribuir sus beneficios, pero nunca de acuerdo al capital aportado. Sin embargo, el segundo grupo está constituido por aquellas entidades que se caracterizan por distribuir su producción de forma gratuita 0 a precios no significativos, por no distribuir sus beneficios en caso de obtenerlos, y por obtener sus recursos a través de donaciones y de la Administración Pública. De acuerdo con estas características, existe un acuerdo universal sobre las principales formas jurídicas que conforman el subsector de no mercado: las asociaciones y las fundaciones de acción social. Estas entidades y su relación con la empresa social son analizadas en los apartados siguientes del presente artículo, los referidos al enfoque del Tercer Sector de Acción Social.

Respecto al sector de mercado, objeto de estudio de este apartado, en España está compuesto por las entidades cuyas formas jurídicas se corresponden con cooperativas, mutualidades, sociedades agrarias de transformación, cofradías de pescadores, sociedades laborales, centros especiales de empleo (CEE) y empresas de inserción (EI); y, en general, también se engloba en este subsector 
cualquier entidad que cumpla con las características descritas anteriormente y que son propias de la economía social ${ }^{1}$ (Monzón et al., 2008).

\section{Enfoque de la economía solidaria}

Partiendo del enfoque de la economía social, la literatura específica también reconoce que sus entidades pasan de estar regidas por unos principios tales como la solidaridad, la reciprocidad, la redistribución y el mercado, a insertarse dentro de la lógica capitalista y a regirse sólo por el principio de mercado (Polanyi, 1944). Este cambio ha dado lugar a que nazca una nueva corriente de autores que reclaman una vuelta a los orígenes de la economía social, a las intenciones reformistas y a los principios sobre los que ésta se sustentaba en sus inicios. Esta nueva doctrina es considerada como una perspectiva propia y diferente a la de la economía social y recibe el nombre de "economía solidaria" (Coraggio, 2011; Guerra, 2013; Martínez y Álvarez, 2008). Tiene su origen en Latinoamérica con Razeto en los ochenta (Razeto, 1984) y en Europa en Francia con Laville en los noventa (Laville, 1994).

La economía solidaria se refiere a la producción de bienes y servicios de acuerdo con un modelo alternativo de creación y gestión de las actividades empresariales. Se trata de empresas que, tomando como base la necesidad de rentabilidad, eficiencia y sostenibilidad técnica y financiera, colocan a los trabajadores y al interés social en el centro y el fin de su actividad, buscando un equilibrio entre los objetivos económicos, sociales y medioambientales (Da Ros, 2007). Esto comprende las experiencias de las empresas que buscan mejorar la seguridad de los medios de subsistencia, reconocer sus derechos y transformar los patrones de producción y consumo a través de diversas formas de cooperación, la solidaridad y la autoorganización democrática (Pérez de Mendiguren y Etxezarreta, 2015). La economía solidaria también hace hincapié en el lugar de la ética en la actividad económica (Askunze, 2013).

Desde esta concepción, la forma jurídica que adquieran las entidades no es determinante, sino que se entiende que la economía solidaria se sitúa entre el mercado, el estado y la reciprocidad, lo que corresponde a los principios del mercado, la redistribución y la reciprocidad (Laville, 1994).

Según los autores Chaves et al. (2013:129) "en la práctica, las cooperativas y asociaciones son las formas dominantes de esta economía solidaria, aunque rechazan que lo sean todas, excluyendo, por ejemplo, a las cooperativas más institucionalizadas y 'banalizadas por el mercado' precisamente por su escaso carácter transformador social". 


\subsection{Escuela anglosajona}

Según la escuela anglosajona, la empresa social es aquella que lleva a cabo actividades económicas en el mercado para servir a su objetivo social independientemente de su forma jurídica (Nyssens et al., 2006). Al igual que en la escuela europea, surge desde la iniciativa privada para mantener los servicios que el Estado dejó de proveer durante la crisis de los años setenta y ochenta. En este sentido, la empresa social es considerada como parte del fenómeno del entrepreneurship, y es estudiada tanto en las business schools como en las ciencias sociales (Hoogendoorn et al. 2010).

Dentro de la escuela anglosajona encontramos dos subescuelas o enfoques: el de generación de ingresos o Tercer Sector de Acción Social, y el del emprendimiento social

\section{Enfoque del Tercer Sector de Acción Social (TSAS)}

Este enfoque también recibe el nombre de "Non-profit"2 (Powell y Steinberg, 2006; Salamon y Anheier, 1998) y centra su atención, al igual que en la escuela europea, en la organización, entendida como aquella entidad sin ánimo de lucro que lleva a cabo una actividad comercial con miras a obtener unos ingresos destinados a apoyar su misión social (Skloot, 1987; Emerson \& Twersky, 1996). En este sentido, en esta subescuela encontraríamos a las entidades del subsector de no mercado de la economía social.

El tipo de entidades que se incluyen dentro del llamado TSAS son las asociaciones de acción social, las fundaciones de acción social, las fundaciones y Obras Sociales de las Cajas de Ahorros y otras fundaciones con acción social de sociedades no financieras y financieras (Bretos y Morandeira, 2016).

Este tipo de entidades realizan actividades muy heterogéneas, diversas, plurales y diferentes entre sí. Entre las actividades que se recogen en el Código de Actividades Asociativas del Ministerio del Interior, destacando (Monzón et al., 2008): la rehabilitación de adicciones patológicas, las minorías étnicas, los movimientos migratorios, asistencia en general, pobreza y marginación, la atención e inserción de personas con discapacidad así como de personas de difícil empleabilidad, la cooperación al desarrollo y solidaridad internacional, entre otras.

2.- El enfoque Non-profit tiene origen anglosajón y sus entidades más representativas a nivel internacional son las fundaciones y las charities anglosajonas. Aunque pueden participar o no en el mercado, en este artículo nos centramos en aquellas entidades que sí llevan a cabo una actividad comercial para conseguir su objetivo, pero que bajo ningún concepto distribuyen beneficios. 


\section{Enfoque de emprendimiento social}

Esta subescuela se centra en el emprendedor social, entendiendo que son individuos que hacen frente a problemas sociales de una forma innovadora sirviéndose del mundo empresarial. El concepto de emprendedor social ha sido definido en varias ocasiones (Drayton, 2002; Bornstein, 2004; Yunus, 2008; Zahra et al., 2009). Sin embargo, destaca la definición propuesta por Dees (1998), quien define al emprendedor social como aquel agente de cambio que adopta una misión para crear y sostener valor social (no sólo valor privado).

En este sentido, la escuela de emprendimiento social mantiene que la explotación de la oportunidad en emprendimiento social puede hacerse tanto en sectores lucrativos como en sectores no lucrativos o incluso a nivel gubernamental (Austin et al., 2006; Guzmán \& Trujillo, 2008). La cuestión que lo define como tal es su misión social, que debe estar claramente orientada a beneficiar al grupo de atención o a la sociedad en general (Johnson, 2000; Thompson, 2008).

La visión del emprendimiento social como un negocio orientado a la misión es cada vez más común entre las escuelas de negocios y fundaciones, que fomentan ahora métodos de negocio más amplios como vía hacia la innovación social. Varios trabajos destacan que además de suministrar productos y prestar servicios a individuos y comunidades, buscan generar beneficios adicionales como incrementar el capital social y mejorar la cohesión social (Frumkin, 2002; Tuckman \& Chang, 2006; Young \& Salamon, 2002). Esto se consigue a través de la participación cercana y activa de los grupos de interés de la empresa, así como a través de las estructuras y procesos de gobierno, ya que de esta forma se garantiza la integración de las empresas sociales en las comunidades a las que sirven y su responsabilidad hacia ellas (Pearce, 2003). Por consiguiente, en el fondo, de acuerdo con este enfoque, las empresas sociales son negocios orientados al mercado con intereses y actividades comerciales utilizados para lograr beneficios sociales y colectivos (Dart, 2004).

\section{Metodología}

Como ya se ha mencionado, el equipo español del proyecto internacional ICSEM llevó a cabo una primera aproximación para identificar distintos modelos de la empresa social en España sobre la base de los principales sectores económicos en los que las empresas sociales estaban presentes, llegando a unos resultados que no eran del todo concluyentes y que invitaban a nuevas investigaciones utilizando una orientación diferente (Díaz-Foncea et al, 2015).

Este estudio, y dada la ausencia de una teoría previa que pudiese servirnos de guía, se aproxima inductivamente al fenómeno de la empresa social en España. Tras una profunda revisión de la lite- 
ratura sobre las distintas propuestas de modelos de empresa social (Dees 1996,1998; Spear et al. 2009; Teasdale, 2012; Kerlin, 2013, 2015; Gordon, 2015) se determinan los elementos que permiten caracterizar a la empresa social y las distintas tipologías que surgen de su combinación. Este análisis bibliográfico no fue suficiente para identificar las dimensiones que permitieran construir un marco teórico coherente para establecer una tipología de empresas sociales españolas, pero permitió identificar un conjunto de cuestiones que reflejan los principios que rigen la constitución y el comportamiento de las empresas sociales. Así pues, bajo esta nueva óptica, se revisan los resultados del proyecto ICSEM (Millán-Foncea et al., 2017), los cuales ofrecen información complementaria que permite realizar el análisis planteado.

Fruto de este proceso, se identificaron cuatro realidades socio-económicas que reconocían a la empresa social como parte integrante de su ecosistema. Estas eran la economía social, la economía solidaria, el Tercer Sector de acción social y el emprendimiento social. La revisión de la evolución histórica de estas prácticas socio-económicas nos ha permitido identificar las diferentes fases de institucionalización en las que se encuentran y la trayectoria institucional de la empresa social en cada una de ellas.

Para caracterizar las empresas sociales de cada uno de estos sectores nos servimos de los nueve indicadores que definen las tres dimensiones de la empresa social conceptualizada por la red de investigación internacional EMES (Defourny y Nyssens, 2012).

1. Dimensiones económicas y empresariales de las empresas sociales:

- Una actividad continua que produce bienes y/o vende servicios.

- Un nivel significativo de riesgo económico.

- Una mínima cantidad de trabajo remunerado.

2. Dimensiones sociales de las empresas sociales:

- Un objetivo explícito para beneficiar a la comunidad.

- Una iniciativa lanzada por un grupo de ciudadanos u organizaciones de la sociedad civil.

- Una distribución de beneficios limitada.

3. Gobierno participativo de las empresas sociales:

- Un alto grado de autonomía.

- Una facultad de decisión no basada en la propiedad del capital.

- Una naturaleza participativa, que involucra a diferentes partes afectadas por la actividad.

En base a las entrevistas realizadas a las empresas sociales en el proyecto original, se valora si cada tipología de empresa social encontrada en cada una de las cuatro realidades socioeconómicas identificadas cumple cada uno de los indicadores anteriores. Como resultado de este análisis, en el siguiente apartado se propone una matriz en la que cada una de las dimensiones y sus indicadores se presenta en columnas y cada uno de los sectores y sus empresas sociales en filas. 


\section{Resultados}

Siguiendo la metodología expuesta, a continuación se presentan los resultados obtenidos del análisis.

\subsection{Modelo de economía social de mercado}

El estudio de las empresas sociales desde la perspectiva de la economía social de mercado resulta bastante controvertido. La razón es la ausencia de una definición universalmente aceptada de empresa social. El debate sobre si considerar a las entidades de economía social como empresas sociales es incluso más profundo (aunque a veces ambos términos sean utilizados indistintamente) si la definición de empresa social utilizada es diferente a la del tipo ideal propuesta por EMES. No obstante, la literatura académica y los políticos coinciden en el hecho de que hay tres grupos de entidades de economía social que pueden ser identificadas como empresas sociales cualquiera que sea la definición de empresa social elegida (Alvarez, 1999; García \& Esteve, 2007; Melián Navarro et al., 2011):

El primer grupo son las empresas de inserción (El). Son compañías cuyo objetivo es la integración de personas en riesgo de exclusión social a través del mercado de trabajo. Sus orígenes se remontan a los años ochenta. En esa época surgieron varias iniciativas destinadas a la integración de personas con dificultades para la inclusión social a través del mercado laboral. Con el tiempo, estas entidades fueron tomando cada vez más fuerza y en 2007 se aprobó la ley de empresas de inserción (Marcuello et al., 2008). Éstas se constituyen bajo cualquier forma jurídica tradicional y el organismo competente concede el estatus de El siempre y cuando se cumplan las diferentes características definidas por la ley (Ley 44/2007).

El segundo grupo lo componen los Centros Especiales de Empleo (CEE). Éstos también tienen el objetivo de inclusión social a través del mercado de trabajo, pero en este caso se refieren específicamente a personas discapacitadas. Dichos centros también surgen en los años ochenta, pero su institucionalización se produjo antes que el de las El. De esta manera, son reconocidos y regulados por una norma específica en los mismos años ochenta (Ley 13/1982 para la Integración de Personas con Discapacidad), y para ser considerados como tal, deben tener un mínimo de empleados con alguna discapacidad. Cabe poner de manifiesto que esta definición corresponde a las CEE identificados como de la economía social, dado que en función de los objetivos principales y de la titularidad, la literatura los clasifica en CEE de la economía social, CEE de la economía pública y CEE de la economía capitalista (Monzón et al., 2014, Monzón y Herrero, 2016). 
Finalmente, el tercer grupo abarca a las cooperativas de iniciativa social. Estas entidades cumplen todas las características del sector de la economía social (ya que las cooperativas constituyen la forma jurídica más representativa de la economía social) con la característica adicional de estar dedicadas a servir el interés general de la sociedad. En España, éstas surgen al final del siglo XX con el propósito de crear dentro del marco nacional de economía social un modelo organizativo similar al de las cooperativas sociales italianas, las cuales crean valor social y están centradas en la solución de problemas sociales (Díaz Foncea et al., 2012).

Sin embargo, aunque existe un consenso sobre la consideración de estos tres tipos de empresas de economía social como empresas sociales, pueden existir diferencias entre ellas en varios aspectos respecto al concepto de empresa social. En este contexto, y tomando como referencia la definición de tipo ideal de empresa social propuesto por EMES (Defourny \& Nyssens, 2012), es necesario analizar en qué medida estas entidades cumplen las tres dimensiones que dicha definición incluye (DíazFoncea et al., 2015).

Dimensión económica: Todas ellas cumplen el requisito de desarrollar una actividad económica produciendo bienes o vendiendo servicios en el mercado para obtener ingresos, asumiendo el consecuente riesgo, con empleados contratados y compitiendo directamente con las firmas tradicionales. Sólo en el caso de los CEE y las El tienen ayuda económica del gobierno. Los primeros como consecuencia de tener al menos el $70 \%$ de los empleados con alguna discapacidad, y los segundos como consecuencia de adherir a personas en riesgo de exclusión social y desempleo (el número de trabajadores de inserción no debe exceder el 30\% durante los primeros 3 años de vida de la organización, y el $50 \%$ en adelante). Reciben ayuda económica del gobierno para compensar la baja productividad de las personas que trabajan en este tipo de entidades (personas en riesgo de exclusión social 0 personas con discapacidad). Aun así se considera cumplido el requisito riesgo económico porque existen estudios que demuestran que existe una tasa de retorno económico positivo a la Administración Pública y a la sociedad en general, compensando así las ayudas que reciben (FAEDEI, 2015).

Dimensión social: Las tres entidades tienen un objetivo explícito de beneficio a la comunidad y tienen un límite a la distribución de beneficios. Respecto al requisito de ser una iniciativa lanzada por un grupo de ciudadanos u organizaciones de sociedad civil, se pueden encontrar ciertas diferencias entre las tres entidades. Así, mientras las cooperativas de iniciativa social pueden ser creadas por cualquiera de las dos posibilidades, en el caso de las otras dos entidades se pueden hacer algunas puntualizaciones. En el caso de las El, son calificadas como tal siempre y cuando el principal propietario de la empresa (más del $50 \%$ de la propiedad) sea una organización sin ánimo de lucro (cooperativa de iniciativa social, ONG, fundación, incluso el gobierno). En el caso de los CEE, nacen como una iniciativa de asociaciones de familiares de personas con discapacidad. Sin embargo, con el tiempo, han empezado a ser creados por cualquier persona u organización que ve en estas entidades una manera de maximización de beneficios a través de la recepción de subsidios y ventajas de emplear a personas discapacitadas, como son determinados casos de autoridades públicas, fundaciones de grandes bancos y negocios convencionales. 
Gobernanza participativa: Respecto a esta dimensión, El, CEE y cooperativas de iniciativa social tienen un alto grado de autonomía, a menos que pertenezcan al sector público (como es el caso de algunos $\mathrm{CEE}$ ). Sin embargo, mientras las cooperativas son participativas y democráticas en la toma de decisiones por ley, las El y los CEE no lo son. Consecuentemente, debido a que el marco legislativo no considera estos dos requisitos en ambas formas jurídicas, en la práctica podemos encontrar entidades con diferentes grados de participación y democracia.

\subsection{Modelo de la economía solidaria}

La economía solidaria, además de ofrecer principios y enfoques alternativos al funcionamiento de la economía capitalista, propone iniciativas prácticas en todos los subsistemas empresariales (financiación, producción, comercialización y consumo), colocando a las personas y, en general, la sostenibilidad de la vida en el centro y el propósito de su actividad. En la presente sección se describen las diferentes propuestas prácticas de la empresa social existentes en el ámbito de la economía solidaria en España.

En este análisis vamos a considerar como empresas sociales desde la perspectiva socioeconómica solidaria a los que se reconocen a sí mismos como parte de la economía solidaria. En España, este movimiento se ha organizado principalmente a través de REAS -Red de Redes de Economía Alternativa y Solidaria. Es una red nacida en 1995 y que reúne a catorce redes territoriales y cuatro redes sectoriales.

El instrumento que vincula los objetivos de la red REAS con las experiencias y expresiones organizativas de la economía solidaria es la Carta de Principios de la economía solidaria. Esta carta es "un documento que presenta los seis principios que dotan de cohesión política y orientan las prácticas e iniciativas de movimiento" (Askunze, 2013:106).

Estas experiencias prácticas se desarrollan en todos los ámbitos del proceso económico. Su trabajo se basa en principios conformes a la solidaridad, la confianza, la cooperación y el trabajo colectivo. La gente y el bienestar individual y colectivo tienen prioridad sobre la rentabilidad financiera. Con el fin de entender la empresa social en la realidad española de la economía solidaria y discutirlo en relación con las otras realidades socioeconómicas, la examinaremos a la luz de las dimensiones propuestas por Defourny \& Nyssens (2012).

Dimensión económica: Realizan actividades económicas vinculadas a la producción de bienes y servicios beneficiosos, exceptuando aquellos que son perjudiciales para las personas, la sociedad o el medio ambiente. Si nos fijamos en el sector económico en el que las empresas sociales de la economía solidaria española desarrollan su actividad nos damos cuenta de que cubren una amplia gama de áreas del sector servicios como: seguros y servicios financieros, servicios de limpieza, reciclaje y recuperación, recogida y gestión de residuos domésticos e industriales, consultoría empresarial y orga- 
nizativa, servicios sociales y personales, jardinería, hostelería, comercialización de productos alimentarios y textiles y servicios de asesoramiento y formación, principalmente. Debido a la heterogeneidad de la actividad y tipología de las organizaciones, el riesgo económico está condicionado por el entorno en el que operan, algunos de ellos tienen que someterse a las condiciones del mercado de competencia y otros pueden beneficiarse de las ayudas públicas o tienen a la Administración Pública como cliente de sus bienes o servicios

Dimensión social: Éstas son empresas que centran su significado y propósito en el beneficio social, calidad de vida y el desarrollo humano. El interés social es el centro de su actividad, por lo que destierran la maximización del beneficio como objetivo empresarial prioritario. De hecho, desarrollan actividades sin fines de lucro, en las que no existe distribución de beneficios, pues, en el caso de que generen un beneficio económico, lo reinvierten en la generación de nuevos puestos de trabajo, la mejora de su estructura y en proyectos sociales de apoyo. Son iniciativas promovidas por un grupo de ciudadanos y tienen una fuerte relación con la ciudadanía.

Gobernanza participativa: Una de las dimensiones que identifican a la empresa social de la economía solidaria española es que se basa en la democracia, la autogestión y la colectividad. Estimula la participación democrática en la toma de decisiones económicas y se esfuerza por lograr un sistema de gestión participativa y transparente. Está verdaderamente comprometida con la creación de puestos de trabajo en condiciones dignas, la elaboración de políticas y medidas para promover la igualdad de oportunidades para los trabajadores y el establecimiento de escalas salariales justas y proporcionales. Los integrantes del sector ponen de relieve la importancia de la pertenencia a una red, de hecho, todos ellos son parte de las redes locales, nacionales e internacionales.

\subsection{Modelo del Tercer Sector de Acción Social}

Una de las realidades socioeconómicas más importantes desde la cual se identifican empresas sociales, es el Tercer Sector de Acción Social (TSAS). La realidad del TSAS es considerada una fuente esencial de empresas sociales.

En los últimos años, durante la crisis económica, este sector se ha visto afectado por el incremento de demanda social de sus servicios, dado que la intensidad y prolongación de la crisis ha producido un aumento y empeoramiento de las situaciones de vulnerabilidad social, multiplicándose así las situaciones de exclusión social entre la población española. Se ha incrementado en un $25 \%$ el número de atenciones directas desde 2008, consolidándose el TSAS como un agente cada vez más importante dentro del sistema de bienestar social.

Además, se ha producido una disminución de los recursos económicos de las entidades del sector. En parte debido a la disminución de ayudas procedentes del sector público, desde el 2008 al 2013 se ha visto reducido en un $22,4 \%$. Y también a la disminución de ingresos procedentes del ámbito pri- 
vado, se ha producido un descenso del 11,7\% desde el 2010 al 2013. Esto ha provocado que se produzca un cambio en la estrategia de obtención de ingresos, orientándola hacia la autofinanciación. Autofinanciación procedente de actividades de mercado, que se ha incrementado en un $47 \%$ desde el 2008 al 2013 (Ruiz, 2015; Pape et al, 2016).

Esta situación se podría relacionar con un incremento de empresas sociales procedentes del TSAS, ya que se identifican como empresas sociales aquellas asociaciones y fundaciones que con una clara orientación hacia la consecución del interés general, orientan su actividad hacia al mercado para obtener la financiación necesaria.

Son entidades que parten de su objetivo social, siendo la orientación hacia el mercado una opción complementaria escogida para solucionar sus problemas de financiación. En cuanto a la organización en la toma de decisiones, por una parte, las asociaciones se rigen por el principio de la democracia, tomando sus principales decisiones a través de su asamblea general. En cuanto a las fundaciones, por definición no tienen por qué cumplir con el principio de democracia por no tratarse de una entidad asociativa como tal, y no tener como órgano de decisión una asamblea general.

Dimensión económica: Las empresas sociales que proceden del TSAS se caracterizan por incipientes, que nacen de la necesidad de captar financiación distinta a la procedente de las ayudas y subvenciones que reciben de las autoridades públicas y de las donaciones privadas, que se ha visto mermada en los últimos años de crisis. Concretamente este tipo de empresas no tiene una producción continua dado que combinan actividades con impacto económico y otras que estarían fuera del mercado. En cuanto a los trabajadores, cuentan con la participación de voluntarios por lo que todo el trabajo no es remunerado. El riesgo económico que tienen es bajo, dado que también obtienen financiación por otros canales sin riesgo, como son las ayudas o subvenciones.

Dimensión social: Estas entidades tienen por definición un claro objetivo social, dirigido a la consecución del bienestar general. Además, por su propia naturaleza jurídica (asociaciones y fundaciones) se rigen por el Principio de No Distribución de Beneficios, por lo que la distribución de beneficios sí que está limitada. Este tipo de entidades suelen ser promovidas por grupos de ciudadanos con vocación de solucionar determinados problemas sociales y algunas veces los gobiernos, cuando se trata de servicios públicos, también alientan a la creación de dichas entidades. Y por último decir que sí que se les asocia un alto compromiso con la transformación social, dado que son entidades que quieren introducir los valores de solidaridad e igualdad en distintos y variados ámbitos del sistema social.

Gobernanza participativa: Las decisiones no se toman en base al capital, sino que son las personas las que están en el centro de ese proceso de toma de decisiones. En cuanto al nivel de autonomía, estas entidades funcionan con independencia, aunque muchas veces los poderes públicos quieran interferir en su actividad por tratarse de actividades de carácter público. La toma de decisiones en gran medida se rige por el principio de la democracia, ya que las asociaciones por su propia naturaleza deben tomar las decisiones en su asamblea general. En el caso de las fundaciones, depen- 
derá del criterio que se escoja de manera particular, dado que no son entidades de carácter asociativo y es el patronato el que en última instancia toma las decisiones. Para terminar, respecto a la pertenencia a redes, en el caso español, como ya se ha mencionado, existe la Plataforma del Tercer Sector que es una organización representativa paraguas de gran parte de las entidades de acción social españolas, y actúa como interlocutor con los poderes públicos defendiendo sus intereses.

\subsection{Modelo de emprendimiento social}

En los últimos años ha habido un incremento en la literatura sobre el emprendimiento social. El término emprendimiento social se ha consolidado y ha entrado de lleno en la agenda política, económica y académica.

Como señala Hulgård (2010:4) el emprendimiento social "se puede definir como la creación de valor social que se ha producido en colaboración con individuos y las organizaciones de la sociedad civil que se dedican a la innovación social incluyendo normalmente actividad económica".

Y eso es lo que motiva a los emprendedores sociales. Ellos ven un problema social en nuestra sociedad y tratan de arreglarlo usando una actividad económica. Haciendo esto, necesitarán ayuda de otros emprendedores, stakeholders clave o toda la sociedad en general. Por lo tanto, el producto de este proceso innovador es lo que conocemos como empresa social.

Aunque el emprendimiento social ha existido en España durante generaciones, las empresas relacionadas con él eran más enfocadas a la economía social, con asociaciones o cooperativas. No obstante, en la última década, y siguiendo la tendencia global, nuevas empresas sociales (con más denotación empresarial y no tanto de economía social) han emergido en nuestro país.

El crecimiento de las empresas sociales en España se atribuye a tres procesos diferentes (Salinas \& Rubio, 2001:86-87): "1) la tardanza y la lentitud en el desarrollo del Estado del Bienestar y la necesidad de reformas de las políticas sociales, 2) la progresiva externalización de parte de la producción y/o de la gestión de servicios por parte de la Administración Pública, muy especialmente en el ámbito de los servicios sociales, y 3) la expansión en buena parte de la sociedad española de valores asociados a la solidaridad o el voluntariado y, en definitiva, a una sociedad más participativa y activa"

Pero debemos ser cautos sobre lo que llamamos empresa social cuando la idea viene desde la iniciativa del emprendimiento social. Ha habido una eclosión en el número de ideas, start-ups o nuevas organizaciones que se autodenominan "social" porque es lo que está "vendiendo" ahora. Por lo tanto, debemos estudiarlas de acuerdo a la teoría disponible, y en nuestro caso nos centraremos en las dimensiones propuestas por Defourny y Nyssens (2012). 
Dimensión económica: Las empresas sociales en España (cuando son creadas desde un punto de vista de emprendimiento social) cumplen los indicadores propuestos por EMES sobre la dimensión económica. Tienen una actividad económica produciendo bienes o vendiendo servicios para poder ganar sostenibilidad (ya que normalmente no reciben financiación pública). Este tipo de organización tiene un nivel significativo de riesgo económico ya que normalmente son promovidas por un emprendedor que invierte en una idea. Además, afrontan el riesgo de empezar un nuevo negocio en un mercado muy competitivo como cualquier otra empresa. Finalmente, tienen un mínimo de trabajo remunerado que las diferencia de otras iniciativas del sector social como las asociaciones por ejemplo. Como en cualquier otro negocio tendrán un presupuesto para pagar al emprendedor (y a otros posibles trabajadores) tan pronto como tengan beneficios de la actividad económica. Por supuesto que se tiene en cuenta el voluntariado en algunas empresas sociales, pero desde este punto de vista (emprendimiento social) y más si tenemos en cuenta la definición anglosajona, estamos ante un tipo de negocio que quiere dar oportunidades de trabajo a diferentes individuos y el voluntariado no se tiene tanto en cuenta.

Dimensión social: En este tipo de organización se ve claramente el objetivo de beneficiar a la comunidad. Las empresas sociales desde el emprendimiento social nacen con una misión social. Esta misión puede ser explicada de diferentes maneras: para proveer de trabajo a las personas en riesgo o situación de exclusión social, para aliviar los problemas medioambientales o para ayudar con problemas sociales diarios. EMES establece que tiene que ser una iniciativa promovida por un grupo de ciudadanos u organizaciones de la sociedad civil, pero en España las empresas sociales desde el emprendimiento social pueden ser promovidas por una sola persona. Las organizaciones creadas por un grupo de personas u organizaciones están más en línea con la perspectiva de la economía social, causando un punto de ruptura en la aplicación de la definición de EMES. Finalmente las empresas sociales deben de tener una distribución de beneficios limitada. Esto es otro punto de ruptura con la realidad española. Las empresas sociales en España pueden adoptar cualquier forma jurídica. Cuando hablamos de economía social o sector social, en la misma ley se establece que tengan un reparto limitado de beneficios. Pero al no existir ley de empresas sociales en nuestro país, pueden elegir cualquier forma jurídica. Haciendo esto, las empresas sociales desde el emprendimiento social no tienen que dar parte de sus beneficios a una causa social, pudiendo quedárselo todo el emprendedor.

Gobierno participativo: Las empresas sociales tienen un alto grado de autonomía. Como se ha dicho, teniendo en cuenta el punto de vista del emprendimiento social, estas organizaciones pueden hacer rendición de cuentas a sus clientes para ser más transparentes, pero no de manera obligatoria hacia las administraciones públicas. Y el emprendedor tiene toda la autonomía disponible. En la definición de EMES también hay un poder de decisión que no se basa en la propiedad del capital. Esto es más problemático ya que dependiendo de la forma jurídica que se elija, esto será más fácil o no. En España hay algunos ejemplos de empresas sociales que garantizan el empleo a personas con discapacidad, pero ellos no tienen el poder de decisión que tiene el emprendedor o la junta de gobierno. Una solución sería la combinación de la empresa social con una estructura más adhocrática para que todos pudieran participar más fácilmente. Por último, un carácter participativo, que invo- 
lucra diversas partes que se ven afectadas por la actividad. Esto sería más fácil de lograr porque las empresas sociales normalmente se ven ayudadas la sociedad civil. Ellos comparten sus experiencias en reuniones, conferencias o de manera online por lo que la sociedad civil se puede sentir más comprometida. Todo esto dependerá del tipo de organización, si se trata de una cooperativa, el gobierno será más participativo que si se trata de una forma jurídica más capitalista. Así que este punto también puede representar un punto de ruptura en la realidad española.

\subsection{Comparación entre modelos}

En la siguiente tabla se recoge de una manera sistematizada y comparada la información recogida en el análisis de cada uno de los modelos a los que ha dado lugar la investigación. Concretamente se identifica de manera gráfica si cada modelo cumple o no los criterios, para así poder determinar las diferencias entre cada uno de ellos.

Los modelos que más se ajustan a los criterios de la definición de empresa social de EMES son el de economía solidaria y dentro del de economía social, las cooperativas de iniciativa social. Ambos cumplen todos los criterios, por lo que se puede considerar que dichas entidades se pueden catalogar como empresa social "ideal".

En una situación intermedia estaría el modelo del Tercer Sector de Acción Social, con una orientación social y de gobernanza participativa, pero con una dimensión económica incipiente. Este tipo de entidades empieza a desarrollar la actividad económica para poder financiar su organización con una orientación clara a la consecución de un objetivo social.

En tercer lugar se encuentra el modelo que siguen las El y los CEE, dentro del modelo de la economía social de mercado. Son entidades que cumplen casi todos los criterios excepto en el caso de la participación y la democracia, esto es debido a la naturaleza de los colectivos de trabajadores que se integran dichas entidades, muchas veces no cualificados para tomar cierto tipo de decisiones.

$Y$ finalmente, el modelo de emprendimiento social es el que menos requisitos cumple. Se observa una fuerte orientación al mercado con un objetivo social, pero en muchas ocasiones no se garantiza el cumplimiento de los criterios de la dimensión social y de gobernanza, perdiendo así garantías de que se pueda considerar a cualquier entidad de ésta realidad socioeconómica una empresa social acorde al concepto de EMES. 


\section{Tabla 1. Modelos de empresa social en España según las distintas realidades socioeconómicas}

\begin{tabular}{|c|c|c|c|c|c|c|c|c|c|c|}
\hline \multicolumn{2}{|c|}{ MODELOS SEGÚN LA } & \multicolumn{3}{|c|}{ DIMENSIÓN ECONÓMICA } & \multicolumn{3}{|c|}{ DIMENSIÓN SOCIAL } & \multicolumn{3}{|c|}{ GOBERNANZA } \\
\hline \multicolumn{2}{|c|}{$\begin{array}{l}\text { REALIDAD SOCIO- } \\
\text { ECONÓMICA }\end{array}$} & \begin{tabular}{|c|} 
Producción \\
Continua
\end{tabular} & $\begin{array}{l}\text { Trabajo } \\
\text { pagado }\end{array}$ & $\begin{array}{l}\text { Riesgo } \\
\text { económico }\end{array}$ & $\begin{array}{l}\text { Objetivo } \\
\text { social } \\
\text { explíito }\end{array}$ & $\begin{array}{l}\text { Distribución } \\
\text { limitada de } \\
\text { beneficios }\end{array}$ & $\begin{array}{c}\text { Iniciativa de } \\
\text { la sociedad } \\
\text { civil }\end{array}$ & $\begin{array}{c}\text { Alto grado } \\
\text { de } \\
\text { autonomía }\end{array}$ & $\begin{array}{c}\text { Naturaleza } \\
\text { participativa }\end{array}$ & $\begin{array}{c}\text { Toma de } \\
\text { decisiones no } \\
\text { basada en }\end{array}$ \\
\hline \multirow{3}{*}{ 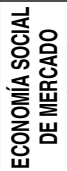 } & Els & $\sqrt{ }$ & $\sqrt{ }$ & $\sqrt{ }$ & $\sqrt{ }$ & $\sqrt{ }$ & $\sqrt{ }$ & $\sqrt{ }$ & $x$ & $x$ \\
\hline & CEES & $\sqrt{ }$ & $\sqrt{ }$ & $\sqrt{ }$ & $\sqrt{ }$ & $\sqrt{ }$ & $\sqrt{1}$ & $\sqrt{2}$ & $x$ & $x$ \\
\hline & $\begin{array}{l}\text { Cooperativas de } \\
\text { iniciativa social }\end{array}$ & $\sqrt{ }$ & $\sqrt{ }$ & $\checkmark$ & $\sqrt{ }$ & $\checkmark$ & $\sqrt{ }$ & $\sqrt{ }$ & $\checkmark$ & $\sqrt{ }$ \\
\hline \multicolumn{2}{|c|}{ ECONOMÍA SOLIDARIA } & $\sqrt{ }$ & $\sqrt{ }$ & $\sqrt{3}$ & $\sqrt{ }$ & $\sqrt{ }$ & $\sqrt{ }$ & $\sqrt{ }$ & $\sqrt{ }$ & $\sqrt{ }$ \\
\hline \multicolumn{2}{|c|}{$\begin{array}{l}\text { TERCER SECTOR DE } \\
\text { ACCIÓN SOCIAL }\end{array}$} & $x^{4}$ & $\mathrm{x}$ & $\mathrm{x}$ & $\sqrt{ }$ & $\sqrt{ }$ & $\checkmark$ & $\sqrt{5}$ & $\sqrt{6}$ & $\checkmark$ \\
\hline \multicolumn{2}{|c|}{ EMPRENDIMIENTO SOCIAL } & $\sqrt{ }$ & $\sqrt{ }$ & $\sqrt{ }$ & $\sqrt{ }$ & $x$ & $x$ & $\sqrt{ }$ & $x$ & $x$ \\
\hline
\end{tabular}

1. En el caso de los CEE de la Economía Pública y la Economía Capitalista, no se cumple el requisito.

2. Existen algunos $C E E$ de titularidad pública.

3. Determinadas entidades dependen de ayudas públicas y de las decisiones de la Administración Pública como cliente.

4. Son entidades que empiezan de una manera incipiente a incorporar actividad económica (en todos sus aspectos), con el tiempo pasarán a cumplir los criterios de la dimensión económica.

5. Muchas veces los poderes públicos quieren interferir en su actividad por ser entidades de utilidad pública.

6. Las asociaciones por naturaleza cumplen el criterio, pero las fundaciones, dependerá del criterio que se escoja de manera particular, ya que es el patronato el que en última instancia toma las decisiones.

\section{Conclusiones}

El objetivo de este trabajo es arrojar luz sobre la realidad de la empresa social en España y establecer una tipología de las mismas. El análisis ha puesto de manifiesto el hecho de que las empresas sociales existen en diferentes realidades socioeconómicas reconocidas en España, como son la economía social de mercado, el Tercer Sector de Acción Social, la economía solidaria y la emprendimiento social, cada una de las cuales alberga un modelo diferente de empresa social. 
Cuando nos referimos a la empresa social desde el punto de vista de la economía social de mercado, nos encontramos con que hay tres realidades diferentes que deben ser consideradas: los Centros Especiales de Empleo, las empresas de inserción y las cooperativas de interés social.

Las empresas sociales que se identifican dentro de la economía social de mercado cumplen claramente los criterios establecidos dentro de la dimensión económica como en la social, mientras que los de gobernanza, depende de la forma jurídica que adopte cada empresa. Las cooperativas por su naturaleza adoptan un proceso de participación y toma de decisiones democrática y en el caso de las El y los CEE dependerá de la voluntad de los dirigentes, aunque en parte quede justificado por la naturaleza y características específicas de las personas que se integran dentro de ellas.

En este sentido, la Ley Española de economía social (2011) se refiere en su preámbulo a la Carta de Principios de la economía social de CEP- CMAF3 y Monzón (2006) considera la democracia interna como discriminante decisiva para la inclusión de una organización sin ánimo de lucro en el ámbito de la economía social. Por lo tanto, cuando se consideran las empresas sociales desde la perspectiva de la economía social, la dimensión de gobernanza es la que marca la diferencia, junto con la distribución limitada de beneficios.

Sin embargo, desde la economía solidaria, el debate sobre el cambio social, la construcción de modelos económicos alternativos, las alternativas al capitalismo o las formas de combatir la pobreza y la exclusión social son el foco central del pensamiento y la reflexión. La empresa social se concibe como parte de un movimiento social que reúne a organizaciones y actividades que están generando un pensamiento y una práctica solidaria y alternativa. Así que, además de la importancia de la dimensión social y de gobierno, lo que marca la diferencia en la empresa social cuando se analiza dentro del contexto de la economía solidaria es su misión de transformación social, entendida como una implicación política o la búsqueda de un cambio de paradigma económico y social.

Las empresas que surgen en el Tercer Sector de Acción Social se caracterizan por cumplir con los factores de la dimensión social y de gobernanza en mayor medida, mientras que los factores económicos se cumplen con menor intensidad, dependiendo del grado de orientación al mercado que se haya adquirido. El cumplimiento de la dimensión social se justifica tanto por su clara orientación hacia el interés general, ya que son entidades por definición de interés general, así como por regirse por el Principio de No Distribución de Beneficios. Y la dimensión de la gobernanza con carácter democrático se cumple plenamente por parte de las asociaciones por su naturaleza asociativa, y en el caso de las fundaciones dependerá de la voluntad de sus dirigentes

La empresa social del sector del emprendimiento social está concebida como un negocio con un propósito, que es resolver un problema social. La principal diferencia con el resto de realidades de empresa social es que su identidad como un negocio, la parte orientada al mercado, es muy fuerte,

3.- Déclaration finale commune des organisations européennes de l'Économie Sociale, CEP-CMAF, 20 juin 2002. 
por los indicadores que lo definen como tal son los que realmente marcan la diferencia con el resto de entidades. El cumplimiento de la dimensión social dependerá del grado de cumplimiento que en cada caso se produzca del objetivo social, dado que su forma jurídica no lo garantiza ni existe ninguna limitación en cuanto al reparto de beneficios. El cumplimiento de la dimensión de gobernanza, al igual que con la dimensión social, dependerá de la voluntad de los emprendedores, dado que nada impide que la decisión se base en la propiedad del capital.

Por lo tanto, podemos concluir que esta investigación pone de manifiesto que en el caso español el objeto de estudio varía dependiendo del enfoque que se utilice para examinar la realidad de empresa social. Este resultado está en línea con el estudio de Defourny y Nyssens $(2012,2016)$ donde distinguen diferentes escuelas de pensamiento a escala internacional sobre la empresa social. Por lo que una primera conclusión es que, a pesar de las particularidades que puedan existir, el caso español no es una excepción en el ámbito internacional de la realidad de la empresa social.

Esta situación puede ser explicada como consecuencia del fenómeno de la globalización. Teniendo en cuenta el avance que han experimentado las tecnologías y las comunicaciones, las distintas iniciativas empresariales con una misión social que nacieron en partes diferentes del mundo motivadas por situaciones diferentes (América y Europa), se han propagado superando fronteras y filtrándose en otros territorios diferentes a aquellos en los que se generaron.

Por otro lado, cabe señalar como una limitación del estudio que, aunque la mayoría de autores consideran a la economía solidaria como un enfoque con entidad propia que constituye una vuelta a los orígenes de la acción solidaria de la que surgió la economía social (Levesque et al., 1989; Defourny \& Develtere, 2009; Laville \& García Jané, 2009), hay otros autores que consideran a la economía solidaria como una prolongación de la economía social (Jeantet, 2001), la llaman la "Nueva Economía Social" (Coque \& Pérez, 2002). Asimismo, algunos afirman que la economía solidaria forma parte del paraguas de la economía social (Pérez de Mediguren et al., 2009). En otros casos, se podrían considerar a la economía social y a la economía solidaria complementarias (Lipietz, 2001), ya que la economía social pondría las reglas del juego mientras que la economía solidaria establecería los valores sobre los que actuar (Coraggio, 2011). De ahí la locución de "economía social y solidaria" (Sajardo \& Chaves, 2006). En este sentido, la consideración de los dos enfoques de forma separada podría verse solapada, ya que, por ejemplo, las empresas de inserción formarían parte de ambos enfoques. Habría entonces que plantearse la posibilidad de unificar ambas perspectivas en el análisis de las empresas sociales, lo cual está previsto realizar en futuras investigaciones.

Otra limitación del estudio radica en que las empresas sociales entrevistadas en el estudio original, se eligieron en base al criterio del sector económico. Por lo que es necesario abordar un nuevo estudio eligiendo la muestra y la metodología de análisis en base a la perspectiva de la realidad socioeconómica que se describe en este artículo. Esta aproximación a la realidad objeto de estudio es debida a que el tema de análisis ha sido escasamente estudiado y, por tanto, no existen teorías formales o sustanciales sobre las que realizar un análisis empírico, por lo que el avance en el conoci- 
miento se produce utilizando diferentes enfoques y los resultados son consecuencia de todos los procesos previos.

Este es un trabajo inicial, que propone una tipología a la que se ha llegado siguiendo un procedimiento inductivo. El equipo tiene diseñadas futuras investigaciones para, a través del análisis de los datos obtenidos mediante encuestas y entrevistas en profundidad a un mayor número de las entidades que representan los modelos de cada una de las realidades socioeconómicas identificadas, demostrar la validez de los modelos planteados.

\section{Bibliografía}

ALTER, K. (2007): "Social enterprise typology", Virtue Ventures LLC, 12, 1-124.

ÁLVAREZ, M.I. (1999): "Las Empresas de inserción social como nueva forma de organización empresarial. Especial referencia a su régimen jurídico", CIRIEC-España, Revista de Economía Pública, Social y Cooperativa, 31, 47-84.

ARRIETA, F.J. (2014): "Concreción de las entidades de la economía social", REVESCO, Revista de Estudios Cooperativos, 116, 33-56.

ASKUNZE, C. (2013): "Más allá del capitalismo: alternativas desde la Economía Solidaria", Documentación social, 168, 91-116.

AUSTIN, J., STEVENSON, H. \& WEI-SKILLERN, J. (2006): "Social and commercial entrepreneurship: The Same, different or both?", Entrepreneurship Theory and Practice, 30(1), 1-22.

BACQ, S. \& JANSSEN, F. (2011): "The multiple faces of social entrepreneurship: A review of definitional issues based on geographical and thematic criteria", Entrepreneurship \& Regional Development, 23(5-6), 373-403.

BATTILANA, J. \& LEE, M. (2014): "Advancing research on hybrid organizing-Insights from the study of social enterprises", The Academy of Management Annals, 8(1), 397-441.

BESHAROV, M.L. \& SMITH, W.K. (2014): "Multiple institutional logics in organizations: Explaining their varied nature and implications", Academy of Management Review, 39(3), 364-381.

BRETOS, I. \& MORANDEIRA, J. (2016): "La Economía Social ante la actual crisis económica en la Comunidad Autónoma del País Vasco", REVESCO, Revista de Estudios Cooperativos, 122, 7-33. 
CHAVES, R. et al. (2013): "La economi a social en clave internacional. Cuantificacion, reconocimiento institucional y visibilidad social en Europa, Iberoamérica y norte de África", REVESCO, Revista de Estudios Cooperativos, 112, 122-150.

CORAGGIO, J.L. (2011): "Economía social y solidaria. El trabajo antes que el capital”. En: Acosta, A. \&Martínez, E. (Eds.), Quito: Abya Yala.

DA ROS, G.S. (2007): "Economía solidaria: aspectos teóricos y experiencias", Unircoop, 5 (1), 9-27.

DART, R. (2004): "The legitimacy of social enterprise", Nonprofit management and leadership, 14(4), 411-424.

DAVISTER, C., DEFOURNY, J. \& GRÉGOIRE, O. (2004): "Work integration social enterprises in the European Union: an overview of existing models", RECMA, Revue Internationale de l'Economie Sociale, 293, 24-50.

DEES, J.G. (1996): Social enterprise spectrum: Philanthropy to commerce, Cambridge, MA: Harvard Business School Press.

DEES, J.G. (2001): The meaning of social entrepreneurship, https://entrepreneurship.duke.edu/newsitem/the-meaning-of-social-entrepreneurship/

DEES, J.G. \& ANDERSON, B.B. (2006): "Framing a theory of social entrepreneurship: Building on two schools of practice and thought", Research on social entrepreneurship: Understanding and contributing to an emerging field, 1(3), 39-66.

DEFOURNY, J. \& NYSSENS, M. (2012): "El enfoque EMES de la empresa social desde una perspectiva comparada", CIRIEC-España, Revista de Economía Pública, Social y Cooperativa, 75, 734.

DEFOURNY, J. \& NYSSENS, M. (2017): "Fundamentals for an international typology of social enterprise models", VOLUNTAS: International Journal of Voluntary and Nonprofit Organizations, 28(6), 2469-2497.

DÍAZ-FONCEA, M. et Al. (2017): Social Enterprise in Spain: A Diversity of Roots and a Proposal of Models, ICSEM Working Papers, No. 29.

DÍAZ, M., MARCUELLO, C. \& MARCUELLO, CH. (2012): "Empresas sociales y evaluación del impacto social", CIRIEC-España, Revista de Economía Pública, Social y Cooperativa, 75, 179-198.

DOHERTY, B., HAUGH, H. \& LYON, F. (2014): "Social enterprises as hybrid organizations: A review and research agenda", International Journal of Management Reviews, 16(4), 417-436.

DRAYTON, W. (2002). "The citizen sector: Becoming as entrepreneurial and competitive as business", California management review, 44(3), 120-132.

EMERSON, J. \& TWERSKY, F. (Eds.) (1996): New social entrepreneurs: The success, challenge and lessons of non-profit enterprise creation, The Homeless Economic Fund, the Roberts Foundation. 
FAEDEI (2015): Memoria Social 2015 Empresas Inserción Laboral, FAEDEl, Madrid.

FRUMKIN, H. (2002): "Urban sprawl and public health”, Public health reports, 117(3), 201-217.

GALASKIEWICZ, J. \& BARRINGER, S.N. (2012): "Social enterprises and social categories". In: Gridon, B. \& Hasenfeld, Y. (Eds.), Social Enterprises, Palgrave Macmillan, London.

GARCÍA, A. \& ESTEVE, M. (2007): "Las Empresas de Inserción en España: normativa y características", CIRIEC-España, Revista de Economía Pública, Social y Cooperativa, 59, 153-178.

GLEMAIN, P., BIOTEAU, E. \& DRAGAN, A. (2013): "Les finances solidaires et l'économie sociale en roumanie: une réponse de «proximités» à la régionalisation d'une économie en transition?", Annals of Public and Cooperative Economics, 84(2), 195-217.

GONÇALVES, C.P., CARRARA, K. \& SCHMITTEL, R.M. (2016): "The phenomenon of social enterprises: are we keeping watch on this cultural practice?", Voluntas, 27(4), 1585-1610.

GORDON, M. (2015): "A Typology of Social Enterprise Traditions", ICSEM Working Papers, No. 18.

GRANADOS, M.L., HLUPIC, V., COAKES, E. \& MOHAMED, S. (2011): "Social enterprise and social entrepreneurship research and theory: A bibliometric analysis from 1991 to 2010", Social Enterprise Journal, 7(3), 198-218.

GUERRA, P. (2013): "Legislaciones sobre ESS en América Latina y el Caribe, Temas, 75, 45-52.

GUZMÁN VÁSQUEZ, A. \& TRUJILLO DÁVILA, M.A. (2008): "Emprendimiento social -Revisión de literatura", Estudios Gerenciales, 24(109), 105-125.

HOOGENDOORN, B., PENNINGS, E. \& THURIK, R. (2010): "What do we know about social entrepreneurship: An analysis of empirical research", ERIM Report Series, Reference No. ERS-2009044-ORG.

HULGÅRD, L. (2010): "Discourses of social entrepreneurship-Variations of the same theme?", EMES Working Papers, No. 10/01.

JOHNSON, S. (2000): Literature review on social entrepreneurship, Working Paper 1-17, Canadian Center for Social Entrepreneurship.

KERLIN, J. (Ed.). (2009): Social enterprise: A global comparison, Hannover, NH: Tufts University Press.

KERLIN, J. (2013): "Defining Social Enterprise across Different Contexts: A Conceptual Framework Based on Institutional factors", Nonprofit and Voluntary Sector Quarterly, 42(1), 84-108.

LAVILLE, J.L. (1994): L'Économie solidaire. Une perspective internationale, París: Desclée de Brouwer.

MAIR, J., MAYER, J. \& LUTZ, E. (2015): "Navigating institutional plurality: Organizational governance in hybrid organizations", Organization Studies, 36(6), 713-739.

MARCUELLO, C., BELLOSTAS, A. \& MARCUELLO, CH. (2008): Informe sobre las Empresas de Inserción en España, CIRIEC-España, Valencia. 
MARTÍNEZ, A. \& ÁLVAREZ, S. (2008): "La economía crítica y solidaria: Perspectivas teóricas y experiencias para la construcción de una economía alternativa." In: The Wordwatch Institute (Ed.), La situación del mundo 2008. Innovaciones para una economía sostenible. Informe anual del Wordwatch Institute sobre el progreso hace una sociedad sostenible, Barcelona: CIP-Ecosocial e Icaria, 371-486.

MELIÁN NAVARRO, A., CAMPOS CLIMENT, V. \& SANCHIS PALACIO, J.R. (2011): "Emprendimiento social y empresas de inserción en España. Aplicación del método Delphi para la determinación del perfil del emprendedor y las empresas sociales creadas por emprendedores", REVESCO, Revista de Estudios Cooperativos, 106, 150-172.

MILLER, T., GRIMES, M., MCMULLEN, J., \& VOGUS, T. (2012): "Venturing for others with heart and head: How compassion encourages social entrepreneurship", Academy of Management Review, 37(4), 616-640.

MONZÓN, J.L. \& CHAVES, R. (2012): La economía social en la Unión Europea, Bruselas: Comité Económico y Social Europeo.

MONZÓN, J.L. (2006): "Economía Social y conceptos afines: fronteras borrosas y ambigüedades conceptuales del Tercer Sector", CIRIEC-España, Revista de Economía Pública, Social y Cooperativa, 56, 9-24.

MONZÓN, J.L. (Dir.) (2008): Las grandes cifras de la economía social en España, Valencia: CIRIEC España.

MONZÓN, J.L., ANTUÑANO, I. \& MURGUI, S. (2014): Informe sobre el impacto económico y social de los Centros Especiales de Empleo en España, Resumen ejecutivo, Fundación ONCE.

MONZÓN, J.L. \& HERRERO, M. (2016): "Identificación y análisis de las características identitarias de la empresa social europea: aplicación a la realidad de los Centros Especiales de Empleo de la economía española", CIRIEC-España, Revista de Economía Pública, Social y Cooperativa, 87, 295-326.

NYSSENS, M. (2006): Social enterprise: At the crossroads of market, public policies and civil society, London: Routledge.

PEARCE, J. (2003): Social Enterprise in Anytown, Calouste Gulbenkian Foundation, London.

PAPE, U., CHAVES, R., BENEDIKT, J., PETRELLA, F., PIELINSKI, B. \& SAVALL, T. (2016): "Working under pressure: economic recession and third sector development in Europe", International Journal of Sociology and Social Policy, 36 (7/8), 547-566.

PÉREZ DE MENDIGUREN, J.C. \& ETXEZARRETA, E. (2015): "Economía social y solidaria: desde la negación al reconocimiento mutuo", Revista De Economía Mundial, 40, 123-144. 
PÉREZ DE MENDIGUREN, J. C. ETXEZARRETA, E., y GURIDI, L. (2009). "Economía social, empresa social y economía solidaria: diferentes conceptos para un mismo debate", Papeles de Economía Solidaria, 1, 1-41.

POLANYI, K. (1944): The great transformation, Boston, Beacon Press (ed. Castellano: 2007, La gran transformación. Los orígenes políticos y económicos de nuestro tiempo, Buenos Aires, Fondo de Cultura Económica.

POWELL, W.W. \& STEINBERG, R. (2006): The nonprofit sector: A research handbook, New-Haven, Yale University Press, United States.

RAZETO, L. (1984). Economía de solidaridad y mercado democrático (Vol. 1). Santiago de Chile, Programa de Economía del Trabajo, (PET).

RUIZ, R. (Dir.) (2015): El Tercer Sector de Acción Social en 2015: impacto de la crisis, Plataforma de ONG de Acción Social, Madrid.

SALAMON, L.M. \& ANHEIER, H.K. (1998): "Social origins of civil society: Explaining the nonprofit sector cross-nationally", Voluntas: International journal of voluntary and nonprofit organizations, 9(3), 213-248.

SALINAS, F. \& RUBIO, M.J. (2001): "Tendencias en la evolución de las organizaciones no lucrativas hacia la empresa social", CIRIEC-España, Revista de Economía Pública, Social y Cooperativa, 37, 79-116.

SANTOS, F., PACHE, A.C. \& BIRKHOLZ, C. (2015): "Making Hybrids Work: Aligning Business Models and Organizational Design for Social Enterprises", California Management Review, 57(3), 36-58.

SANTOS, F.M. (2012): "A positive theory of social entrepreneurship", Journal of Business Ethics, 111(3), 335-351.

SKLOOT, E. (1987). "Enterprise and commerce in non-profit organizations". In: POWELL W.W. (Ed.), The nonprofit sector: A research handbook, Yale University Press. United States, 380-393.

SPEAR, R., CORNFORTH, C. \& AIKEN, M. (2009): "The governance challenges of social enterprises: evidence from a UK empirical study", Annals of Public and Cooperative Economics, 80, 247-273.

TEASDALE, S. (2012): "What's in a Name? Making Sense of Social Enterprise Discourses", Public Policy and Administration, 27(2), 99-119.

THOMPSON, J.L. (2008): "Social enterprise and social entrepreneurship: where have we reached?: A summary of issues and discussion points", Social Enterprise Journal, 4(2), 149-161.

TUCKMAN, H.P. \& CHANG, C.F. (2006): "Commercial Activity, Technological Change, and Nonprofit Mission". In: Powell, W.W. y R. Steinberg, (Eds), The Nonprofit Sector: A Research Handbook, $2^{\text {nd }}$ edition, Yale University Press, 629-644. 
VILLAJOS, E. \& SANCHIS, J.R. (2013): "Las empresas sociales y el management social”, Revista vasca de economía social = Gizarte ekonomiaren euskal aldizkaria, 10, 189-216

WRY, T. \& YORK, J.G. (2017): "An identity-based approach to social Enterprise", Academy of Management Review, 42(3), 437-460.

YOUNG, D. \& SALAMON, L. (2002): "Commercialization, social ventures, and for-profit competition". In: L. Salamon (Ed.), The state of nonprofit America, Washington, DC: Brookings Institution Press, 425-448.

YUNUS, M. (2008): Un mundo sin pobreza, las empresas sociales y el futuro del capitalismo, Paidos Ibérica, Barcelona.

ZAHRA, S.A., GEDAJLOVIC, E., NEUBAUM, D.O. \& SHULMAN, J.M. (2009): "A typology of social entrepreneurs: Motives, search processes and ethical challenges", Journal of business venturing, 24(5), 519-532. 